\section{Practical and illustrated summary of updated BI-RADS for ultrasonography}

Jiyon Lee

Department of Radiology, NYU School of Medicine, NYU Cancer Institute, Breast Imaging Center, New York, NY, USA

The American College of Radiology released the fifth edition of the Breast Imaging-Reporting and Data System (BI-RADS) in 2014 (copyright 2013), which includes the expanded second edition of the ultrasound BI-RADS lexicon. This review provides a practical summary of the updated lexicon, including selective illustrations with original clinical images, a discussion of overarching concepts, and examples of current clinical applications.

Keywords: Ultrasonography; Lexicon; BI-RADS

\section{Introduction}

The American College of Radiology (ACR) fifth edition of the Breast Imaging-Reporting and Data System [BI-RADS] Atlas [1] provides essential updating and expansion of the guidebook central to breast imaging. The BI-RADS lexicon frames and details the language that we speak in the field of breast radiology. The first edition of BI-RADS, published in 1992, covered only mammography, the sole breast-specific imaging modality at that time. The 2003 BI-RADS Atlas was the fourth edition for mammography and the first edition for breast ultrasonography (US) and magnetic resonance imaging (MRI). This fifth edition is the second edition for US and MRI.

The goals of BI-RADS are to (1) standardize imaging reporting, (2) reduce confusion in imaging interpretations by providing a common language among radiologists and among imaging practices, (3) enable reliable communication to referring clinicians regarding the significance of findings and management recommendations, and (4) facilitate outcomes monitoring.

Overarching concepts apply to the entire BI-RADS fifth edition. Real cases replaced the diagrams in the early editions. Deliberate efforts to "harmonize" the lexicon across modalities (mammography, US, and MRI) changed terms and definitions; these are highlighted. There is now an intentional uncoupling of the assessment and management recommendation categories for various clinical situations; examples of this are also provided. Specific to-do and not-to-do recommendations are provided to facilitate outcomes monitoring across modalities. New "frequently asked questions" (FAQs) and guidance chapters are replete with practical help, and there are several summary tables, convenient for the workstation.

This article presents the updated lexicon using original case examples to illustrate and summarize selected grouped US concepts along with highlighting the key changes and overarching concepts.

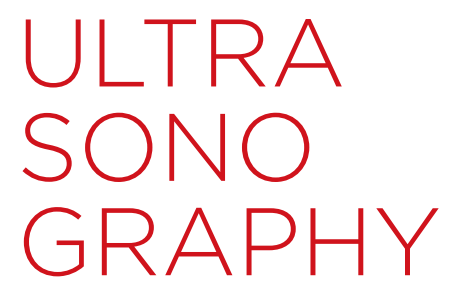

\section{PICTORIAL ESSAY}

https://doi.org/10.14366/usg. 16034 pISSN: 2288-5919 - eISSN: 2288-5943 Ultrasonography 2017;36:71-81

Received: August 9, 2016 Revised: October 2, 2016 Accepted: October 3, 2016

Correspondence to: Jiyon Lee, MD, Department of Radiology, NYU School of Medicine, NYU Cancer Institute, Breast Imaging Center, 160 East 34th Street, 3rd Floor, New York, NY 10016, USA

Tel. +1-212-731-5353

Fax. +1-212-731-5554

E-mail: Jiyon.Lee@nyumc.org

This is an Open Access article distributed under the terms of the Creative Commons Attribution NonCommercial License (http://creativecommons.org/ licenses/by-nc/3.0/) which permits unrestricted noncommercial use, distribution, and reproduction in any medium, provided the original work is properly cited.

Copyright (C) 2017 Korean Society of Ultrasound in Medicine (KSUM)

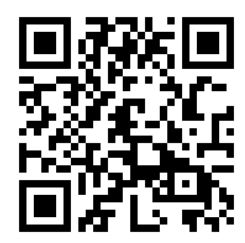

How to cite this article:

Lee J. Practical and illustrated summary of updated BI-RADS for ultrasonography. Ultrasonography. 2017 Jan;36(1)71-81. 


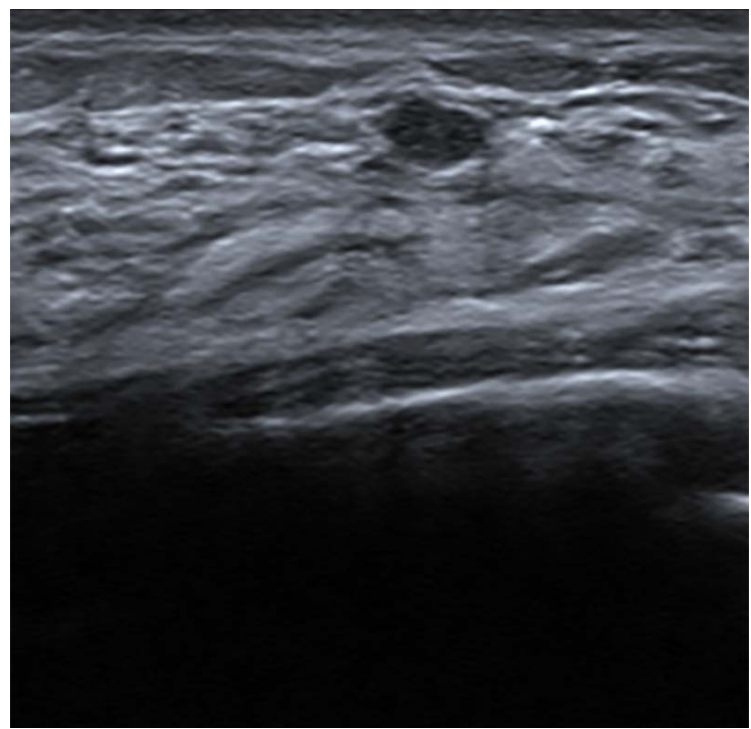

A

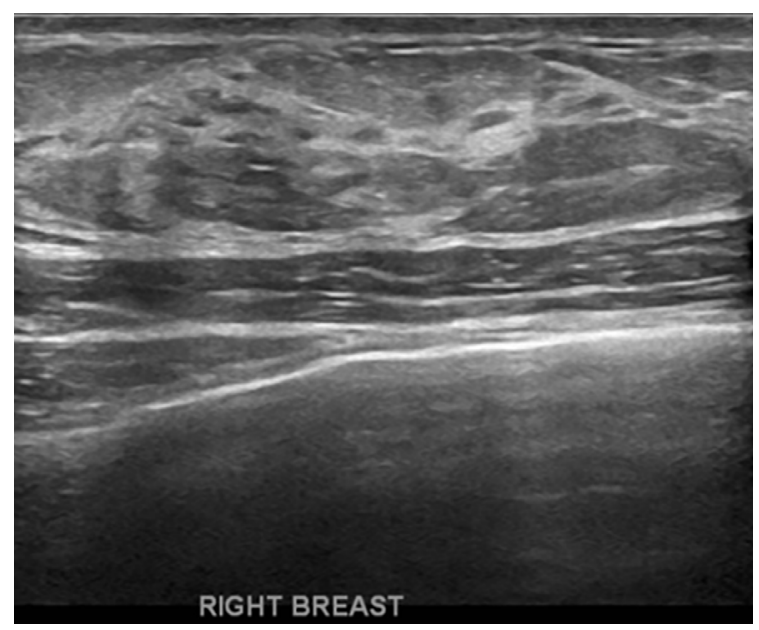

C

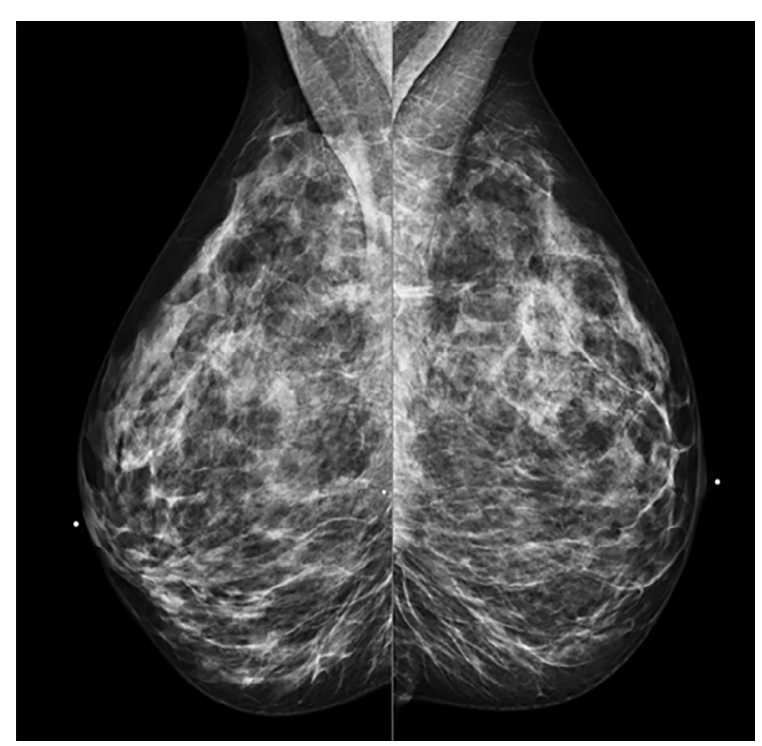

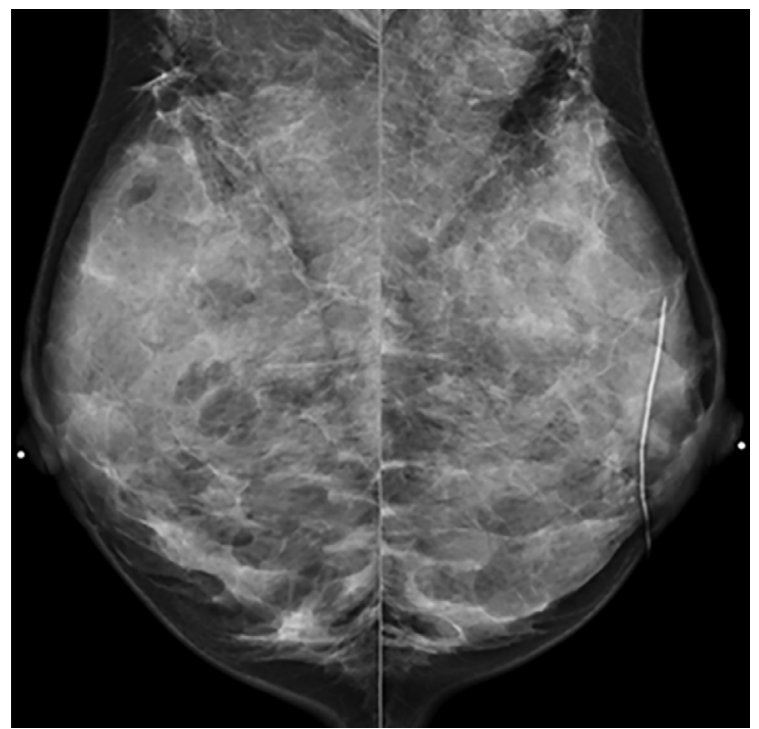

B

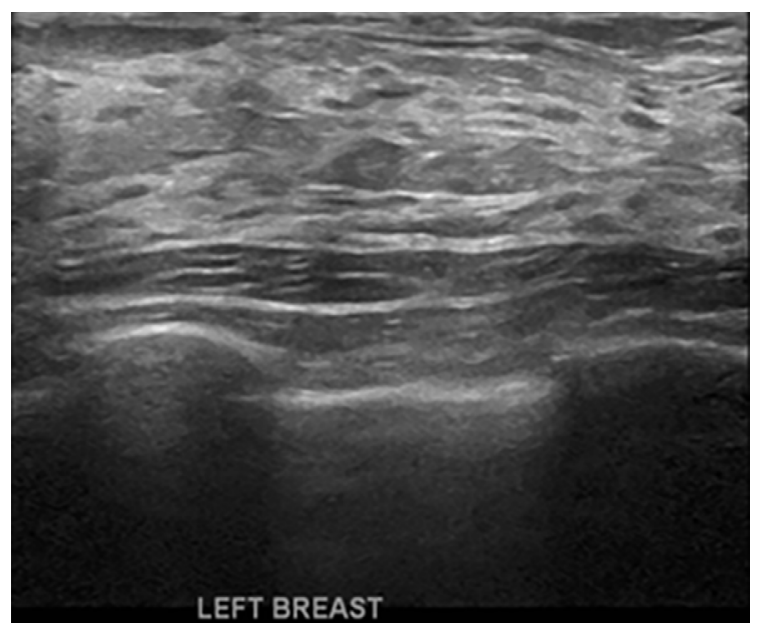

D

Fig. 1. Ultrasonography: tissue composition.

Tissue composition refers to the echogenicity of the background parenchyma, using fat as an internal reference. The dominant tissue echogenicity should in general parallel the mammographic density assessment. The choices are homogeneous background echotexturefat, homogenous echotexture-fibroglandular, and heterogeneous echotexture. This sonogram shows homogeneous echotexture-fibroglandular (A) that corresponds to an extremely dense breast tissue pattern (B) on mammography. Sonograms of a different patient's right (C) and left (D) breasts show the heterogeneous echotexture of fat and parenchyma intermingled, which parallels the mammographic pattern of a heterogeneous density (E). 


\section{US Reporting}

Components of a typical report are listed in the Atlas. Indication for the exam (e.g., diagnostic, palpable complaint, screening recall, short-term follow up, or screening), dates of comparison/correlation exams, pertinent physical exam details, and the scope and technique of the exam (e.g., hand-held, automated US, unilateral/bilateral, complete vs. targeted/limited, technologist/radiologist/both, or special techniques) are expected. New is the recommendation to include a succinct description of the breast composition. Next is a clear description of significant findings, with images to document. For example, at minimum, a negative complete screening US should show all four quadrants and retroareolar regions. Each practice may choose to include axillary views. When mammography and US are jointly performed, composite reports that report in chronological order with one overall BI-RADS assessment are recommended. The most worrisome feature from either or both exams should lead to

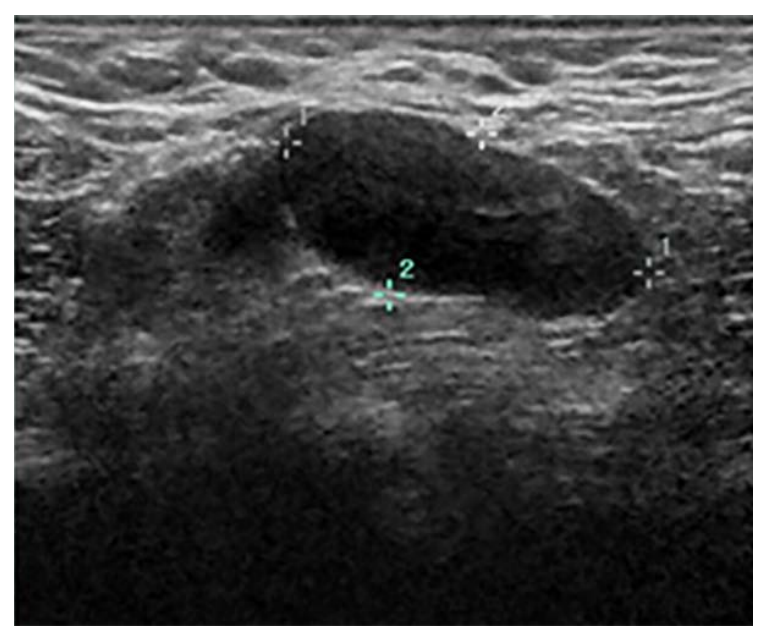

A

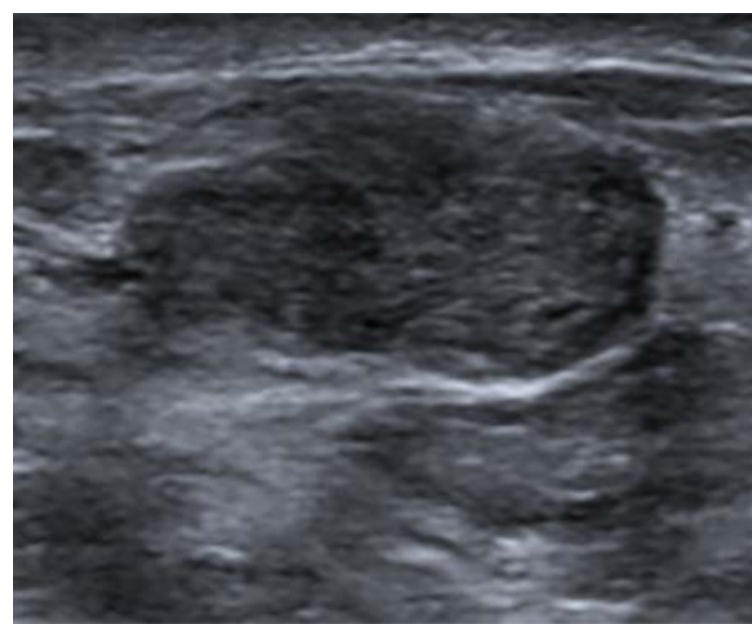

C the final BI-RADS and management recommendation.

\section{US Lexicon Overview}

The following will be presented next, in order as per the Atlas: tissue composition, categories of findings (masses, calcifications, associated features, and special cases), and lexicon classification.

\section{US: Tissue Composition}

Now recommended is a mention of the dominant tissue composition on the screening US (Fig. 1). This should correlate with mammography, if performed. "Homogeneous background echotexture-fat" parallels a predominantly fatty mammogram. "Homogenous background echotexture-fibroglandular" is a corollary for an extremely dense mammography. "Heterogeneous background echotexture" reflects density categories $C$ and $D$, possibly leaning towards a denser tissue pattern.

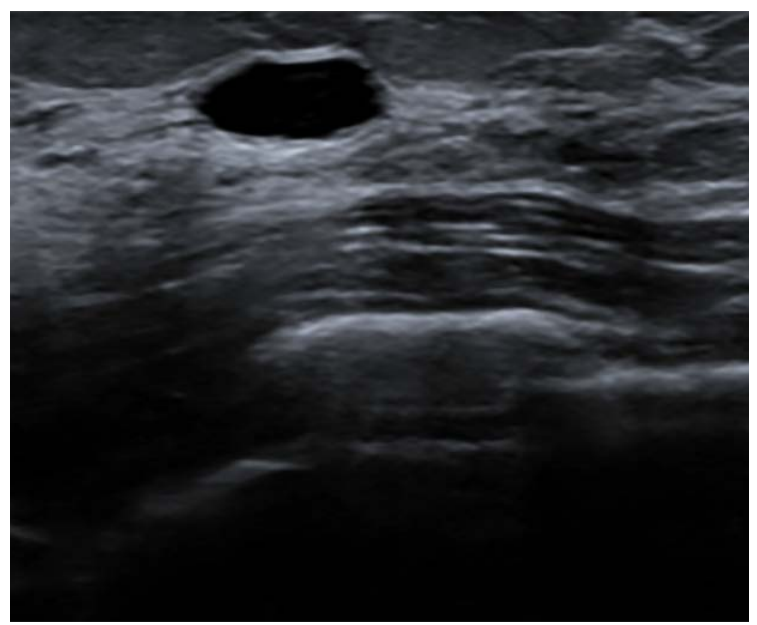

B

Fig. 2. Ultrasonography: mass descriptors-shape.

Three descriptors are in this category of mass shape, similar to mammography. They are (1) oval: elliptical, egg shaped, and having up to 2-3 gentle lobulations; (2) round: spherical, ball shaped, globular; and (3) irregular: neither round nor oval. A-C. These ultrasonographic masses are elliptical, with up to 2-3 lobulations. Therefore, they are called oval. 


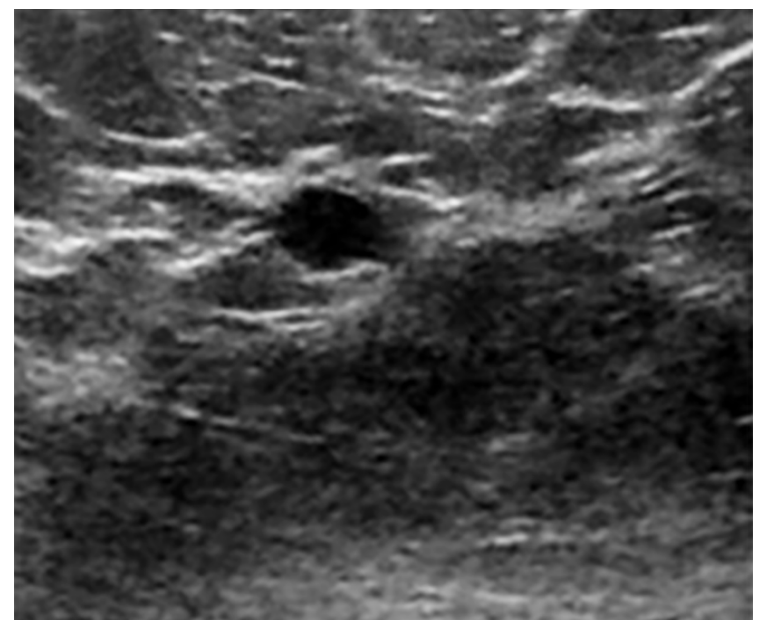

D

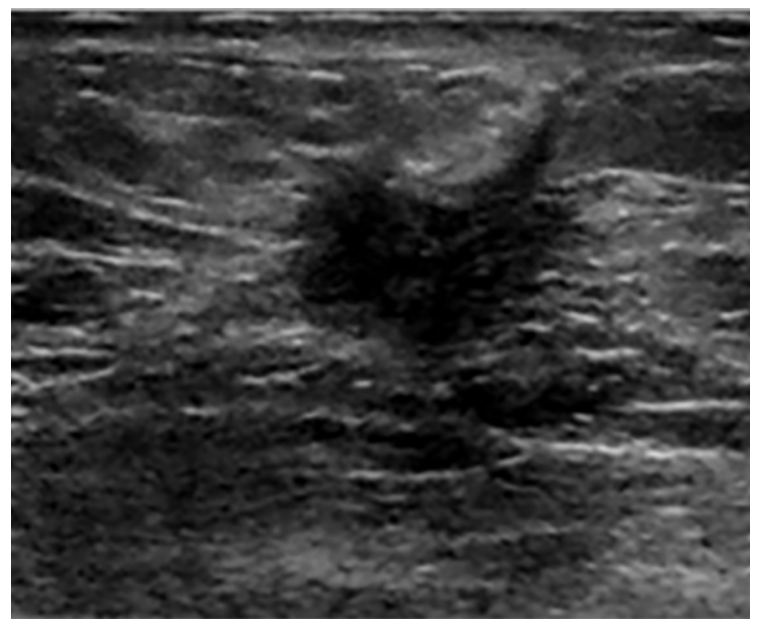

$\mathrm{E}$

Fig. 2. D. This round mass is a simple cyst. E. This mass is irregular, a feature more suspicious but still not specific for malignancy.

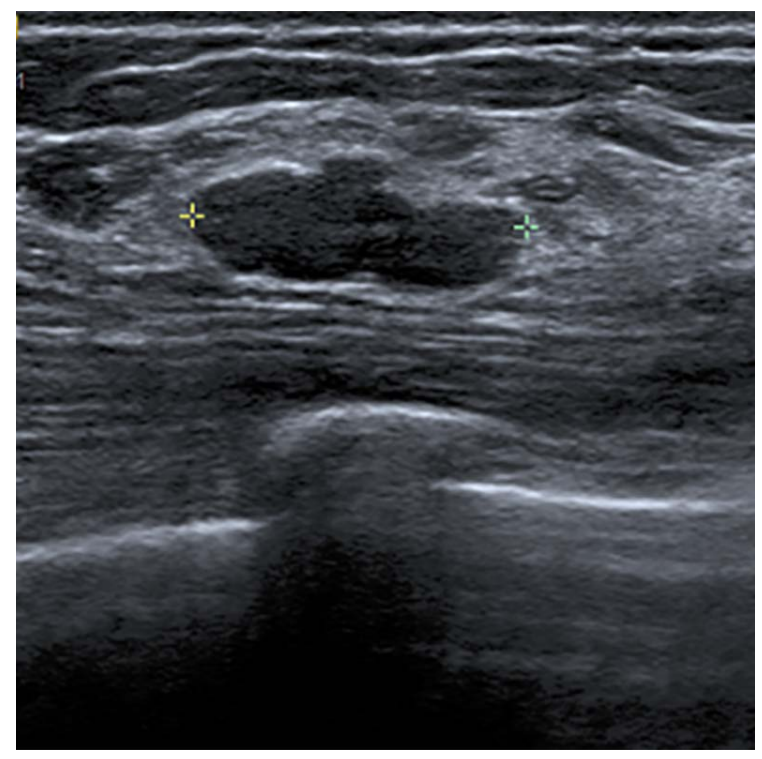

A

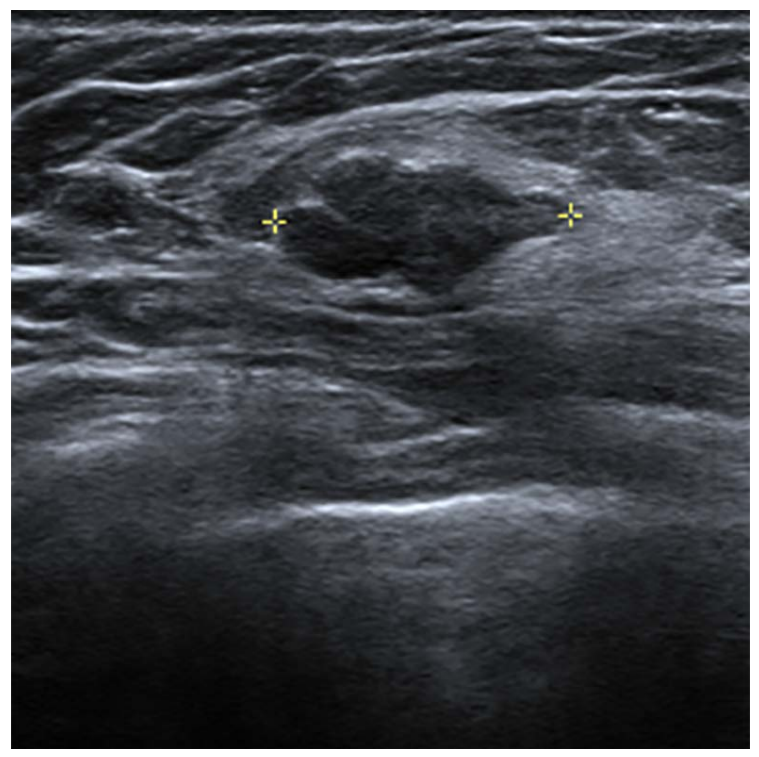

B

Fig. 3. Mass: assessing the shape, margin, and orientation.

A, B. The descriptor "lobulated" is discouraged in the BI-RADS Atlas fifth edition [1], for ease of use rather than for a lack of real examples. Here is one such lesion, shown in orthogonal projections. This is a benign mass, biopsy-proven fibroadenoma and was stable on this patient's serial ultrasonography follow-up. Our differential diagnosis and management recommendation are based on both the mass shape and the other descriptors. It is important to assess all projections and carry out the scan in real time.

In addition to screening US reports, diagnostic cases may occasionally call for a mention of the breast echotexture as a caveat. For example, when seeking a US correlate for a mammographic asymmetry, a hypoechoic or heterogeneous area with subtle shadowing may be visible. This may be suspicious and indeed be the US correlate for the mammographic concern, but the radiologist should beware and be skeptical first. Scanning and assessing broadly is appropriate. If in the rest of the breast(s), there are many areas having a similar ambiguous echotexture with shadowing, then this finding is less specific and reliable, which might undermine diagnostic confidence. Of course, all the breast imaging tools of triangularization and intermodality correlation should be used. An US-guided biopsy is reasonable to test one's suspicion after exercising due diligence. 


\section{US: Findings, Masses}

In a deliberate effort to harmonize with mammography, the mass shape descriptors are not oval (i.e., elliptical, egg shaped, have up to 2-3 gentle lobulations), round (i.e., spherical, ball shaped, globular), and irregular (neither round nor oval) (Fig. 2). This schema is simplified for ease of use, but there are still lesions that do not

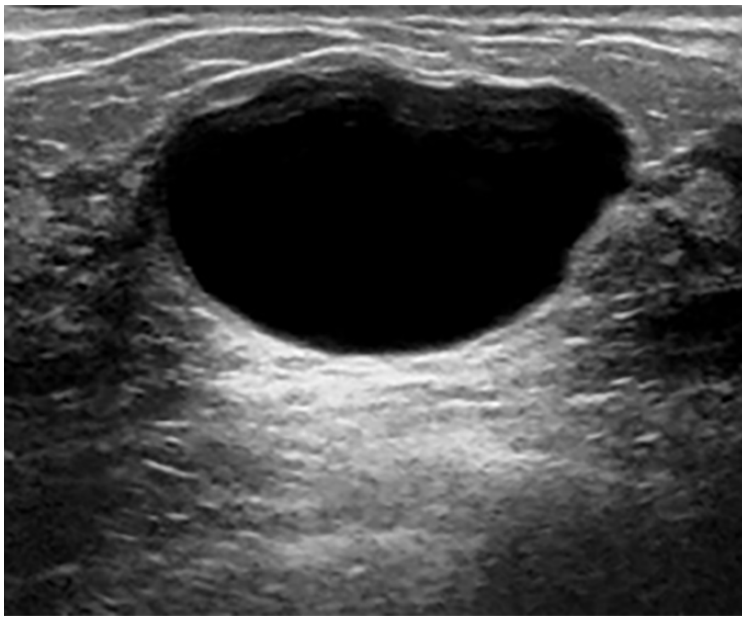

A

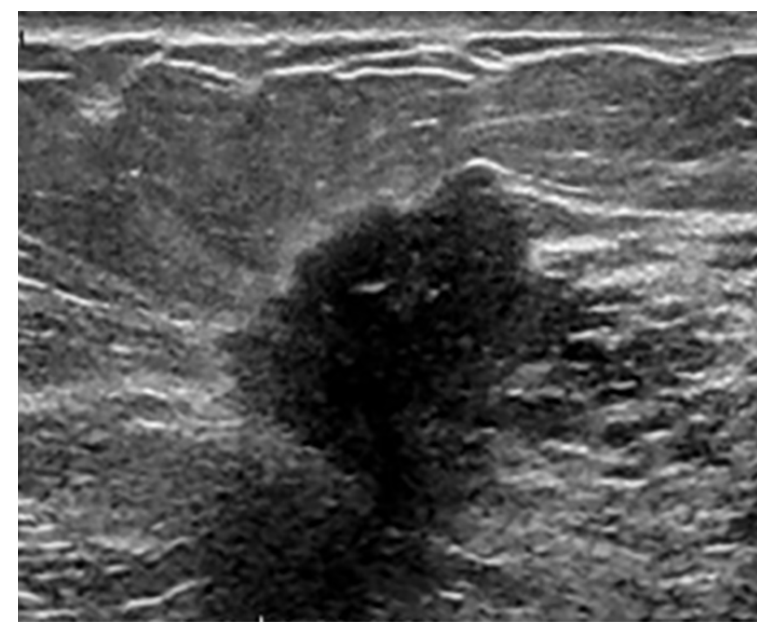

C

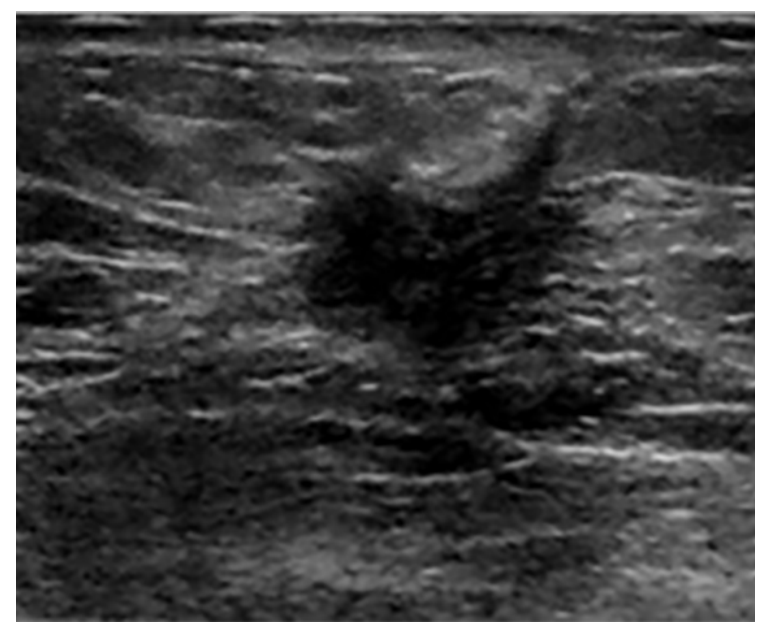

E

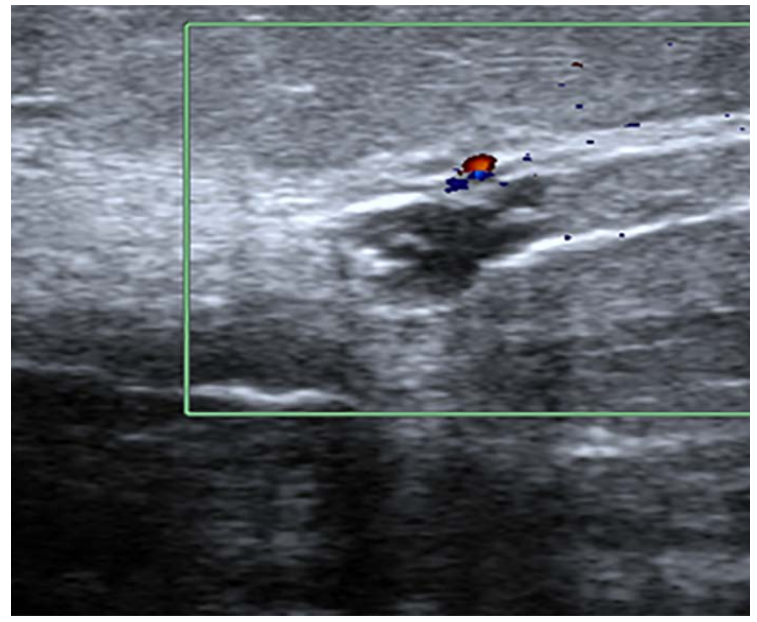

B

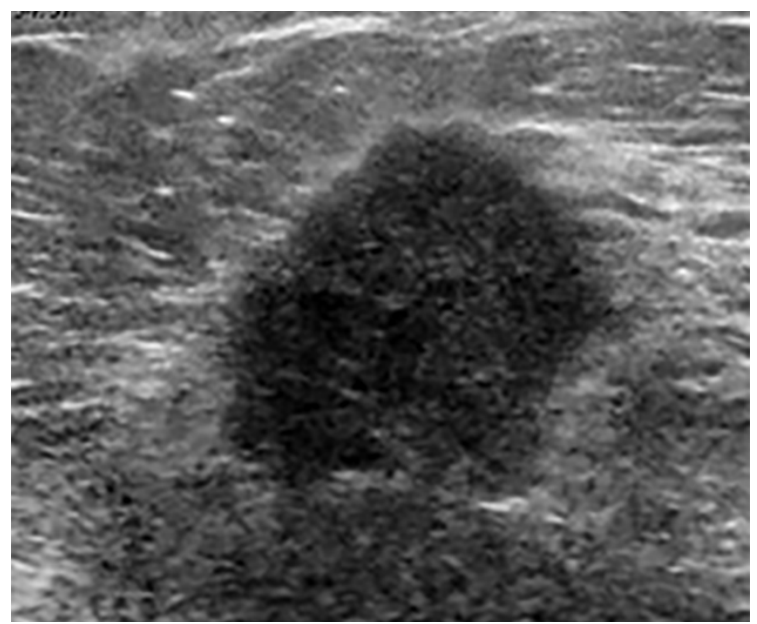

D

Fig. 4. Ultrasonography: mass descriptors-margin.

The branch point for the margin assessment is whether the lesion is circumscribed or not circumscribed. If the lesion is not circumscribed, then the additional descriptors are indistinct, angular (unique to ultrasonography), microlobulated, and spiculated. All margins should be scanned, and the descriptors used in combination as the interpreting radiologist deems adequate. A. This lesion is smoothly marginated and therefore circumscribed, and is a classic simple cyst. B. Another example of a cyst does not show the classic features of a simple cyst. B-E. Noncircumscribed lesions are eligible for additional descriptors, which can and should be used in combination as needed to fully describe the lesion. The examples here show margins that are partially indistinct $(C, D)$, angular $(B, E)$, microlobulated $(C, D)$, and spiculated (E). These terms are used for conveying the degree of imaging suspicion leading usually to a biopsy recommendation. However, the descriptors are not specific enough to avoid a biopsy to confirm suspicion. Lesions $C-E$ are malignancies. 
fall neatly into one of these strict definitions. For example, benignappearing masses with more than three lobulations are common (Fig. 3). The radiologist should use judgment and communicate the details of the case through the prose description.

The descriptors for the mass margin first bifurcate into circumscribed or not circumscribed (Fig. 4). A circumscribed margin suggests but is nonspecific for benignity. The subcategories of a not circumscribed margin are indistinct, angular (unique to US), microlobulated, and spiculated; these suggest "not benign" but do not guarantee malignancy. A full-margin evaluation is important, with a real-time assessment recommended particularly for lesions that are indeterminate or cases in which management is not straightforward. Use these descriptors in combination to fully characterize the margin. Proper usage should lead rationally towards a differential diagnosis and support the overall BI-RADS assessment and management recommendations.

The descriptor orientation, echo pattern, and posterior acoustic features are US specific. These are all secondary to the shape and margin in estimating the likelihood for malignancy. Orientation refers to the lesion's long axis relative to the skin (Fig. 5). Choices are parallel or not parallel, with parallel suggesting a benign etiology. These terms replace the phrases "wider-than-tall" and "taller-thanwide" that are still commonly spoken. By definition, a round mass is neither parallel nor not parallel.

A mass echo pattern relies on fat as the reference echotexture. Choices are anechoic, hyperechoic, complex cystic and solid, hypoechoic, isoechoic, and heterogeneous (Fig. 6). In general, the lesions that we seek are hypoechoic, heterogeneous, or complex cystic and solid.

For posterior acoustic features, choices are no posterior features, enhancement, shadowing, and combined echo pattern. Although posterior acoustic enhancement helps define a classic simple cyst, this feature is not specific for benignity as it can also be seen in lesions such as a metastatic lymph node or metastasis to the breast. In contrast, shadowing is classically associated with a highly suspicious mass, but this suspicious feature is nonspecific and can be seen with benign fat necrosis and stromal fibrosis (Fig. 7).

\section{US: Findings, Calcifications}

The BI-RADS Atlas lists choices as follows: calcifications in a mass, calcifications outside of a mass, or intraductal calcifications. It is not clear whether calcifications outside of a mass can mean calcifications without a mass. Although US is not typically used for screening for calcifications or seeking mammographic calcifications, there are occasions when US is used for correlating with mammographic calcifications. Examples would be calcifications associated with a suspicious mass, in an intraductal location, or difficult to reach stereotactically but biopsiable ultrasonographically if found. Mammography should be used to confirm before reporting calcifications as being present. Some US speckles may not be calcifications at all. If a core biopsy is indicated, specimen radiography should be recommended to confirm calcification sampling. Multiple and different diagnoses can coexist in the same patient, as illustrated in Fig. 8. Sometimes, the calcifications are

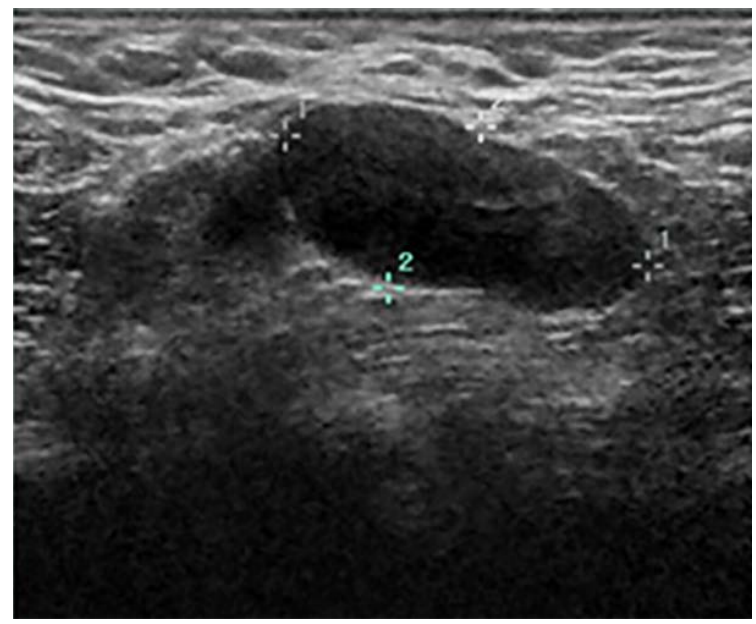

A

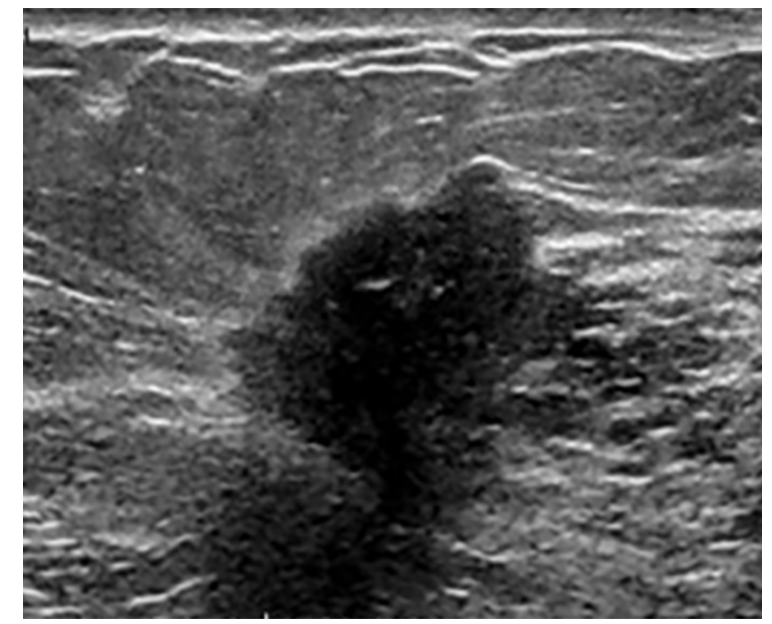

B

Fig. 5. Ultrasonography: mass descriptors-orientation.

Orientation is a descriptor unique to ultrasonography, as it refers to whether the longest axis of a lesion is parallel (i.e., wider-than-tall, horizontal) or not parallel (i.e., taller-than-wide, vertical) to the skin. Not parallel is more concerning than parallel, but still not specific to a malignancy. Round lesions by definition have no longest axis so cannot be considered parallel or not parallel. A. This parallel lesion is a benign fibroadenoma. B. This not-parallel mass is malignant. 


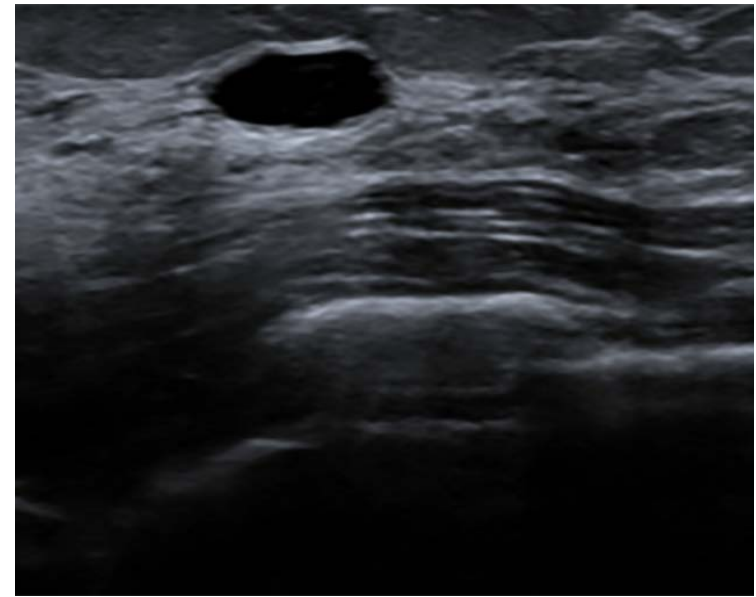

A

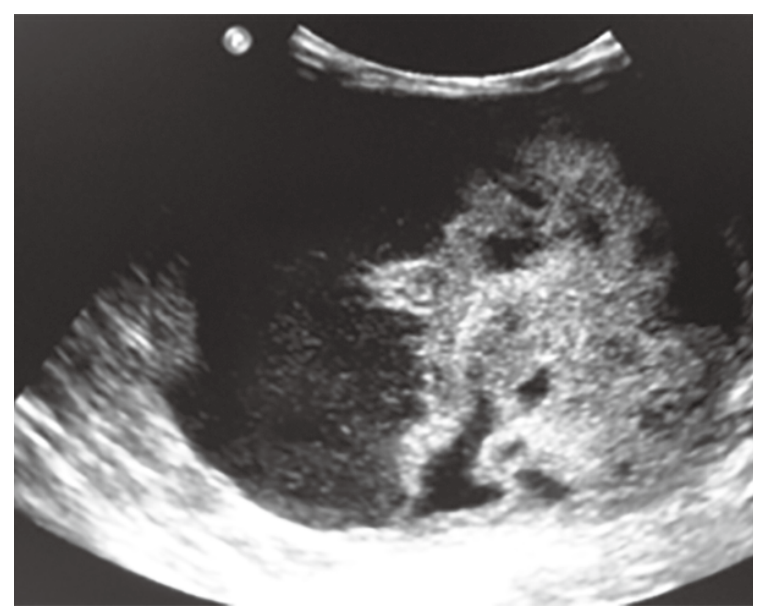

C

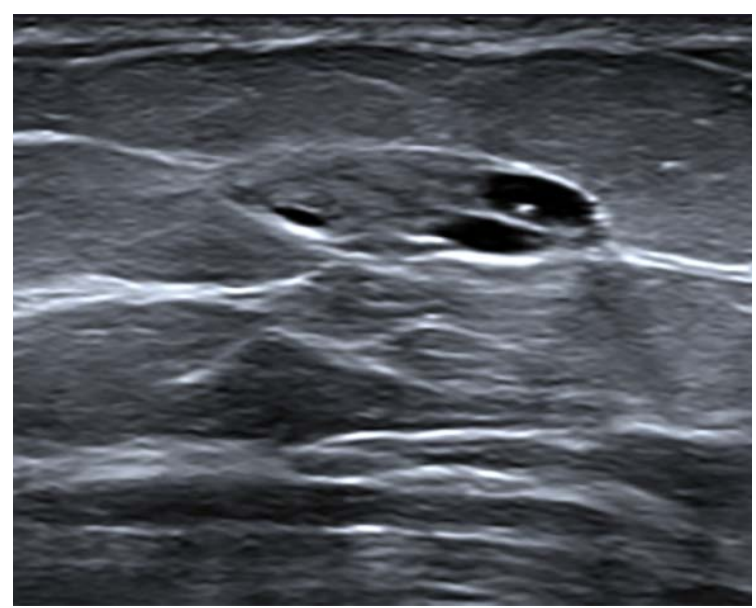

E

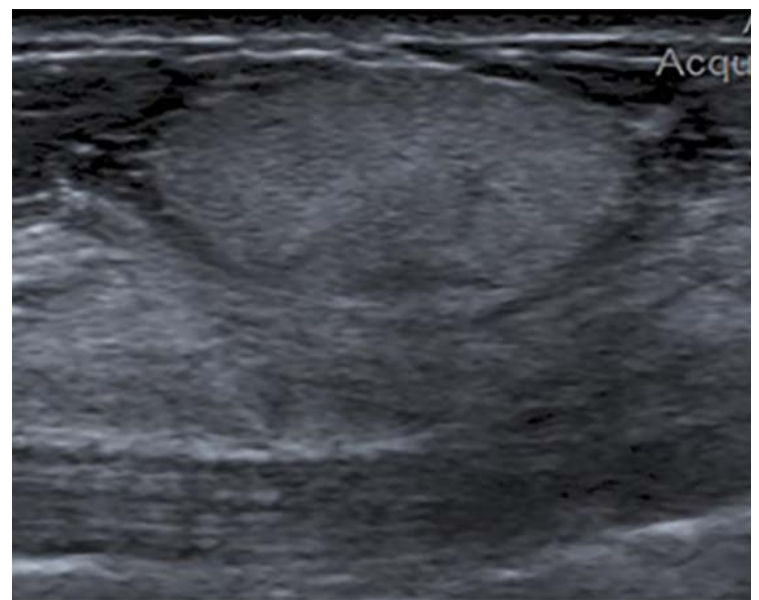

B

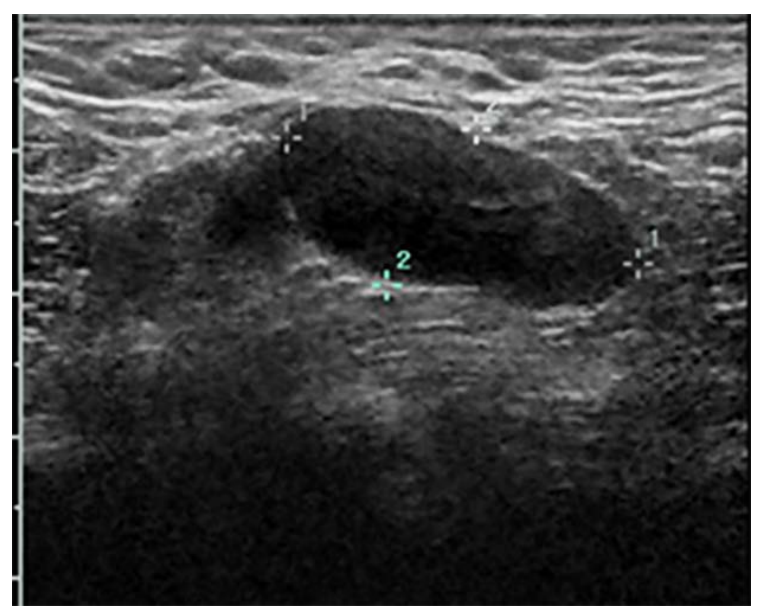

D

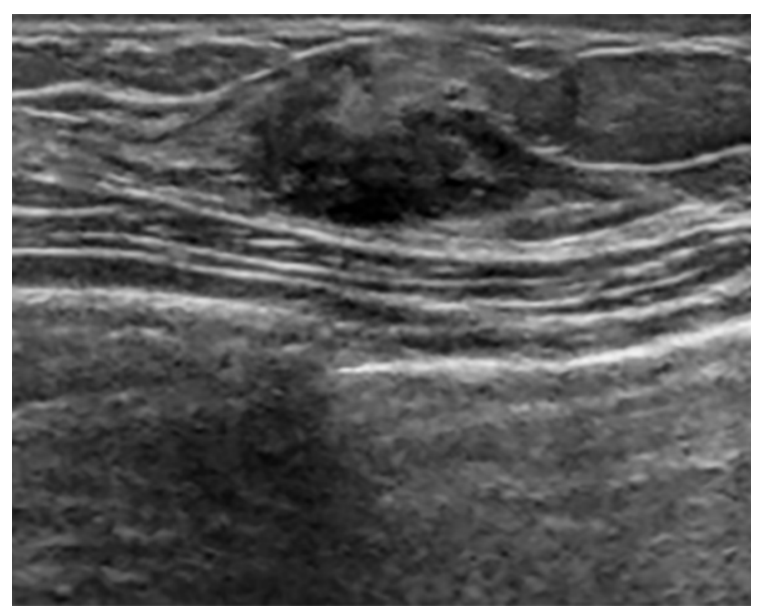

$\mathrm{F}$

Fig. 6. Ultrasonography: mass descriptors-echo pattern.

Echogenicity of the lesion is also a ultrasonography-specific descriptor. Fat is the reference echogenicity. A. This anechoic lesion is a simple cyst. B. An echogenic mass corresponds to a lucent mass on mammography (not shown) and is consistent with lipoma. C. This complex cystic and solid mass is nonspecific and in this case, was diagnosed as an intracystic papillary carcinoma. D. A circumscribed, oval hypoechoic mass was proven to be a benign fibroadenoma. E. This lesion is mostly isoechoic, with small anechoic cystic spaces. This appeared to correlate with a chronic partially lucent mammographic mass (not shown) and is presumed to contain lipids. F. This heterogeneous mass is indeterminate and recommended for a biopsy. 
associated with a mass that is of lower suspicion, and US may confirm or help downgrade mammographic suspicion.

\section{US: Findings, Associated Features}

Please see the BI-RADS atlas for full detailed list. These are architectural distortion, duct changes, skin changes, edema, vascularity, and elasticity assessment. Elasticity is mentioned not as endorsement but rather as acknowledgement that elastography exists and of possible future formal guidance on usage and reporting. The associated features are architectural distortion, duct changes, skin changes, edema, vascularity, and elasticity assessment. Elasticity is mentioned not as an endorsement but as an acknowledgment that elastography exists and of possible future formal guidance on usage and reporting.

\section{US: Findings, Special Cases}

Please refer to BI-RADS atlas for full detailed list and descriptions. Special cases are simple cyst, clustered microcysts, mass in or on skin, foreign body including implants, lymph node-intramammary, lymph node-axillary, vascular abnormalities, postsurgical fluid collection, and fat necrosis. Listed are simple cyst, clustered microcysts, mass in or on the skin, foreign body including implants,

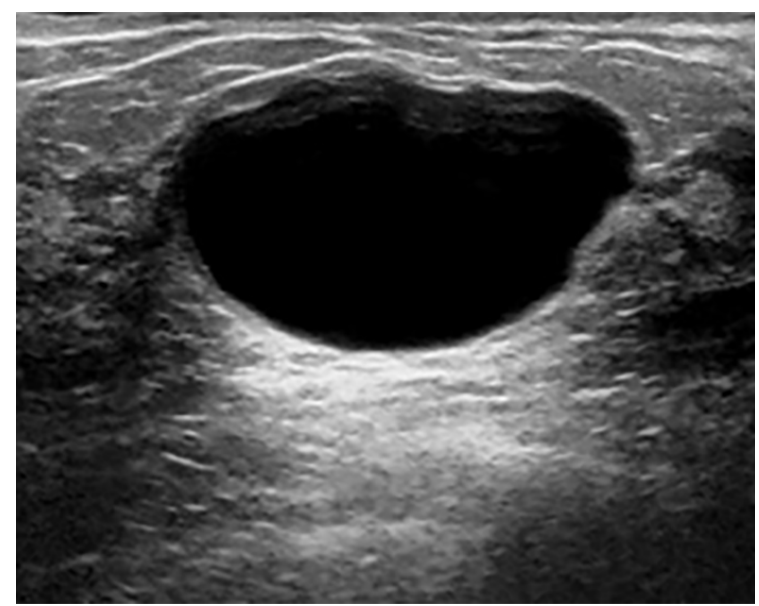

A

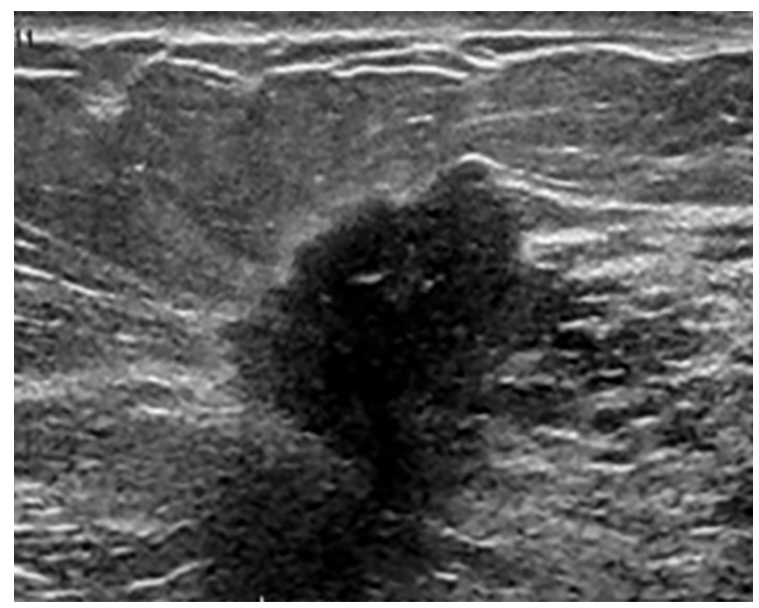

C

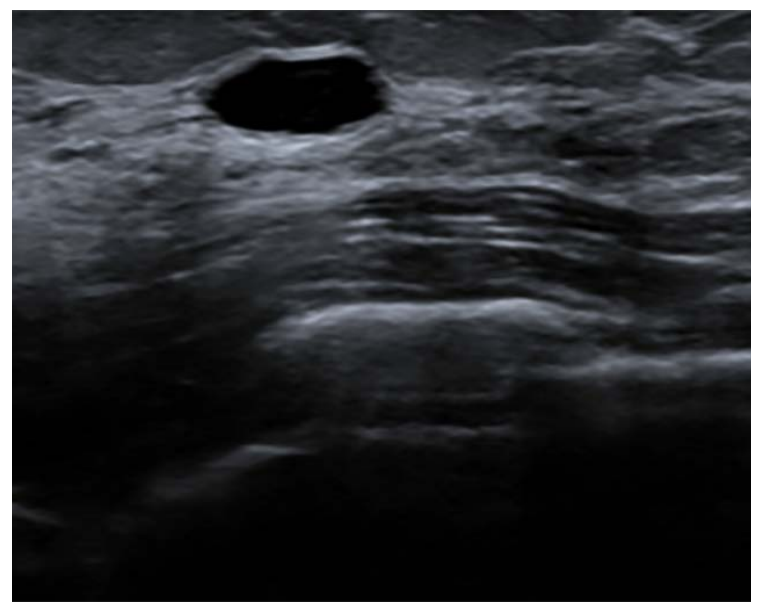

B

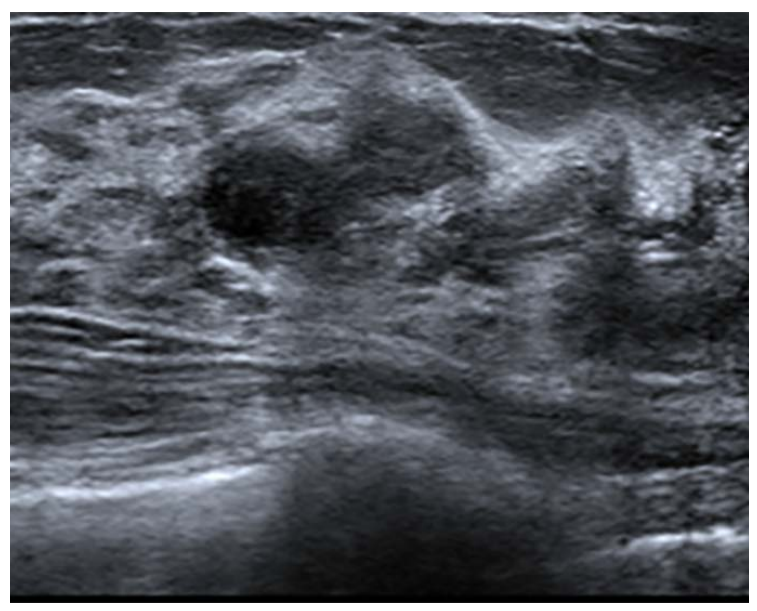

D

Fig. 7. Ultrasonography: mass descriptors-posterior features.

Posterior feature is another ultrasonography-specific descriptor and refers to the attenuation characteristics of a mass relative to its acoustic transmission. The choices are no posterior acoustic features, enhancement, shadowing, or a combined echo pattern. A, B. Lesions that are cysts can show a range of posterior acoustic enhancement that appears as an echogenic column deep to the cysts. Enhancement is not specific to a benign diagnosis; it can also be seen in homogeneous solid lesions such as high-grade carcinomas. C. The malignant lesion demonstrates shadowing deep to it, due to the attenuation of the acoustic transmission. D. This lesion demonstrates a combined echo pattern. Shadowing is associated with fibrosis, with or without carcinoma. Attenuation (shadowing) and enhancement are of a more secondary than primary predictive value, because they are not specific to a benign or malignant diagnosis. 
lymph node-intramammary, lymph node-axillary, vascular abnormalities, postsurgical fluid collection, and fat necrosis.

\section{US: Assessment and Management}

Table 5 in the Ultrasonography section of the atlas maps the BIRADS assessment categories to their likelihoods of malignancy; please refer to that table for more details regarding these and other small changes in language and percentages. Important adjustments now close the percentage gaps in the prior lexicon. The likelihood of cancer for category 3 is clearly stated as $>0 \%$ to $\leq 2 \%$. Even now it is common to hear a reference to BI-RADS 3 to mean a $<2 \%$ chance of cancer. Category 4 is now a $>2 \%$ and $<95 \%$ chance of

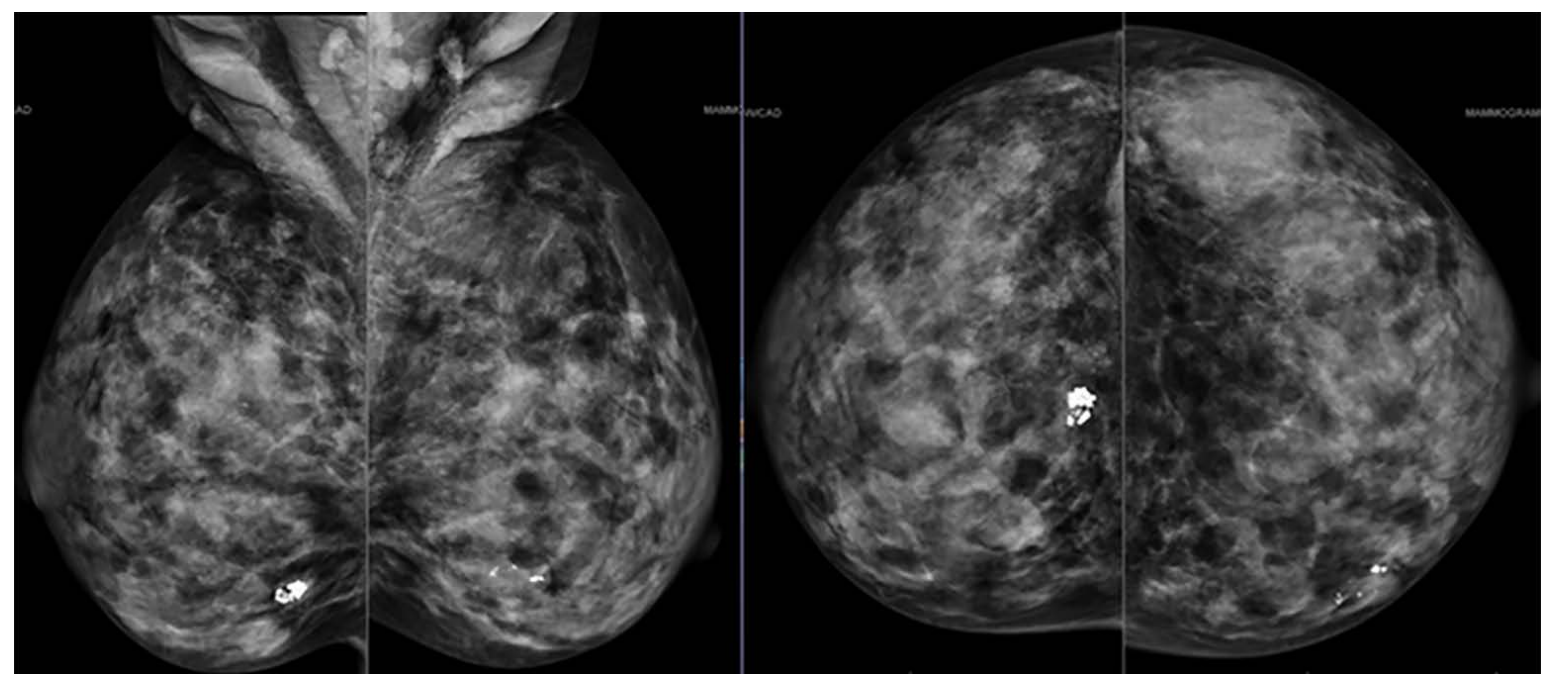

A

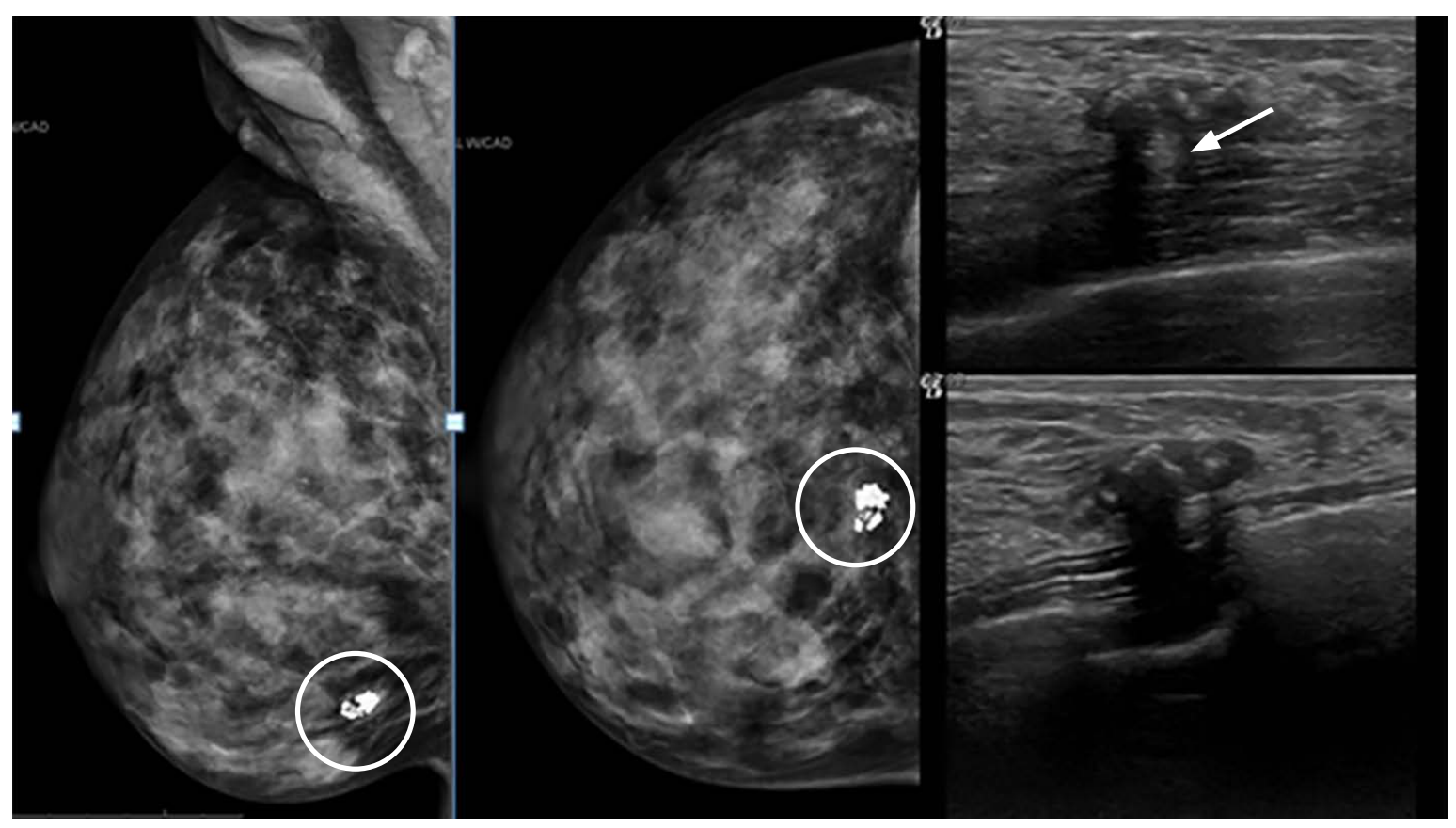

B

Fig. 8. Ultrasonography (US): calcifications on US.

This 37-year-old woman presented with a palpable complaint on the left, and the diagnostic evaluation ultimately revealed three different examples of ultrasonographic masses with calcifications. A. Bilateral mammography shows a dense breast tissue, as expected. Bilateral US reveals both areas of homogenous echotexture-fibroglandular and heterogeneous echotexture. B, C. Coarse calcifications at the right 6:00 axis (circles) (B) and medial left 9:00 axis (circles) (C) are consistent with involuting fibroadenomas, showing varying levels of "popcorn-type" calcifications on the mammography and the correlative US (arrows). 


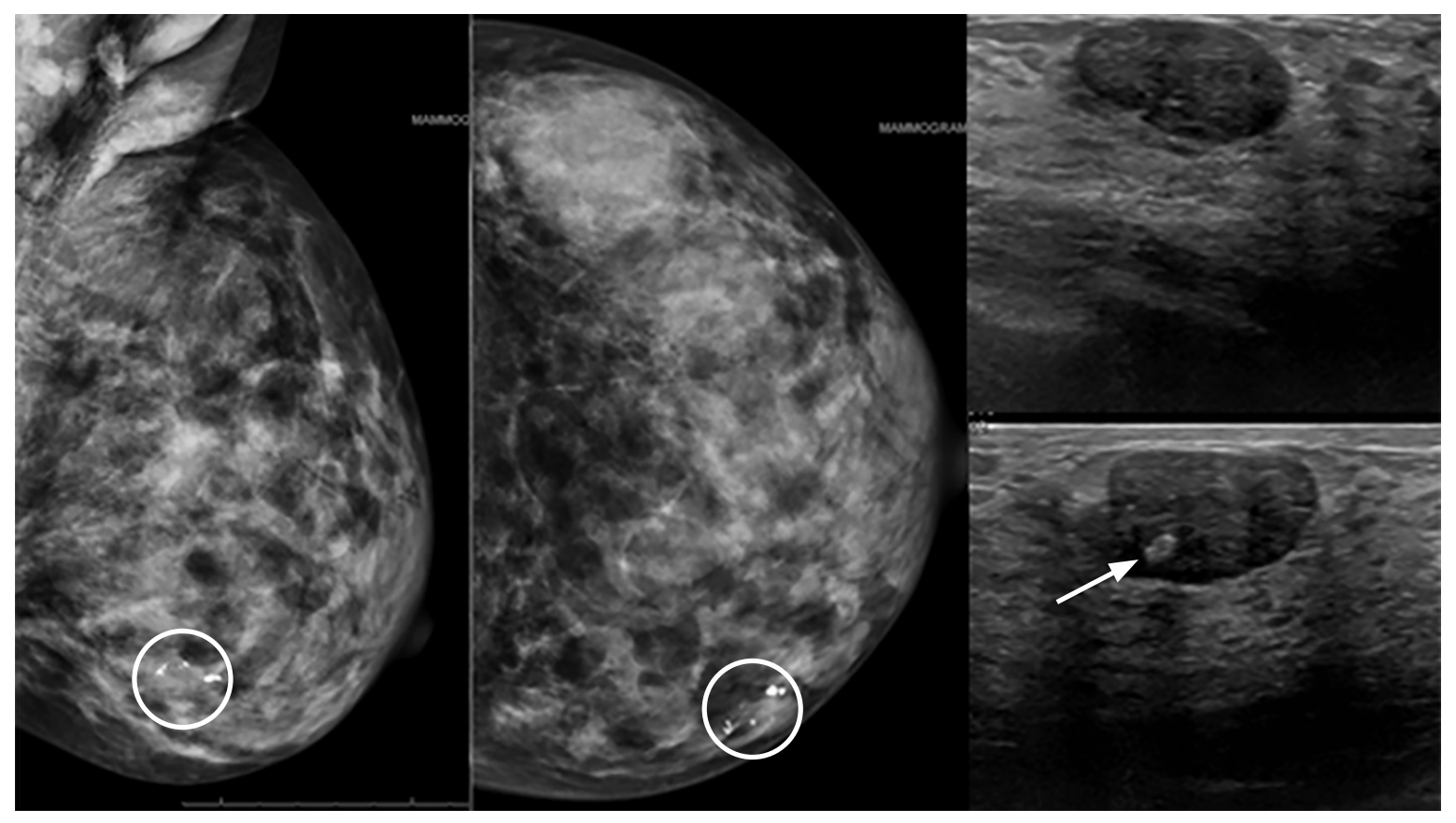

C

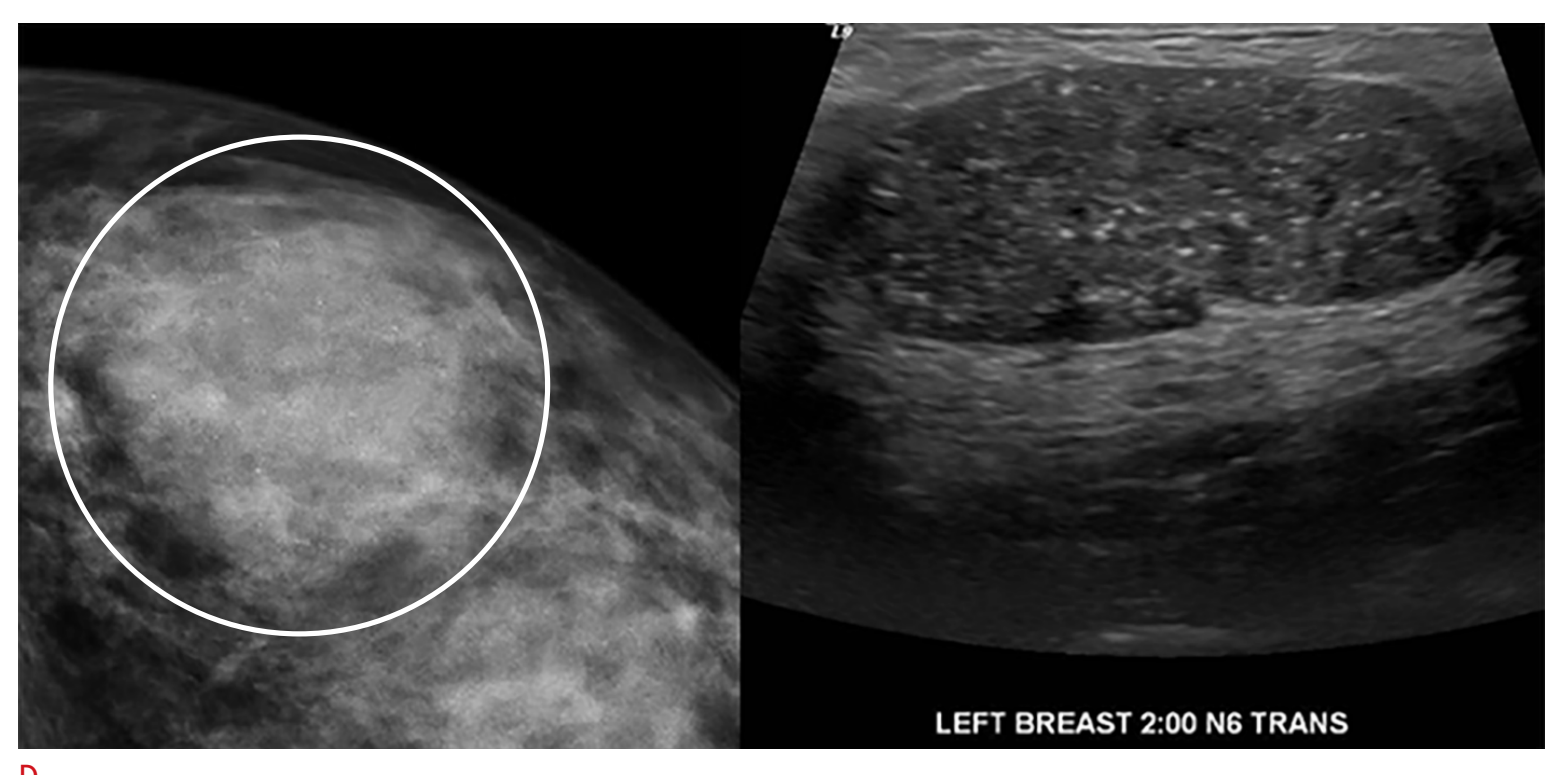

Fig. 8. D. Distinctly different from these lesions, there is a 4-cm isodense mammographic asymmetry at the left 2:00 axis (circle) with associated diffuse faint calcifications. US shows obvious circumscribed, oval, parallel 4-cm mass with speckles of diffuse internal calcifications. The subsequent core biopsy shows a complex fibroadenoma associated with multiple microcalcifications, which is a benign and concordant diagnosis. Not shown here are multiple other smaller masses in both breasts, also likely to be fibroadenomas. Multiplicity of findings is common in the case of breasts. A correlation between mammography and sonography helps ensure proper diagnosis.

cancer, with the subcategories now as follows: $4 \mathrm{~A},>2 \%$ to $\leq 10 \%$ likelihood; $4 \mathrm{~B},>10 \%$ to $\leq 50 \%$ likelihood; and $4 \mathrm{C}_{1}>50 \%$ to $<95 \%$ likelihood. The change in category 4 subdivisions should be noted; "intermediate" has been removed. Category 5 means a $\geq 95 \%$ chance.
Four additional points regarding exam reports are worth mentioning. First, a composite reporting of mammography and US is suggested when both were performed in the same encounter, for instance, for a diagnostic recall from screening or in an evaluation of palpable complaints. The most concerning or worrisome feature 
from either or both modalities should determine the final BI-RADS and management recommendation. In general, BI-RADS 0 cases should be rare to none.

Second is how screening US should be reported. If according to the technologist's static images, there are no findings that the radiologist desires to re-evaluate, then BI-RADS 1 or 2 would be appropriate depending on the findings. However, if the radiologist wishes to scan any lesion with a real-time assessment, even if it is of very low suspicion (e.g., a cyst with debris), then BI-RADS 0 is suggested as the appropriate reading. In such a case, a separate exam and accession for "diagnostic ultrasonography" would proceed. These suggested guidelines aim to (1) "harmonize" with the protocol of a screening mammography and (2) accommodate cases when/where the radiologist is not present. The author imagines a variable uptake of this reporting suggestion that will depend on the practice preferences and staffing.

An additional point relates to when a patient should undergo the next screening test. Until there are standard guidelines, which may not ever happen with US, the reporting radiologist should be clear about her/his own recommendations regarding the mammography and US. "Routine screening" is presumptive language; clear and responsible recommendations are the most helpful to patients and referring clinicians.

The fourth point is the now-official uncoupling of the assessment and management to build in a flexible language for the few occasions that call for it. For example, a 35-year-old woman with an enlarging mass presents for baseline imaging. Dense breast tissue but otherwise nonfocal mammography might lead to bilateral breast US showing multiple cysts, some with debris and one clearly accounting for the palpable site. Notice that this would be BI-RADS 2 overall for benign cysts. However, a therapeutic cyst aspiration would be appropriate to offer as an additional line in the impression, for example, "Therapeutic cyst aspiration is offered, if desired." along with a sentence to the effect of "Screening mammography recommended at the next clinically appropriate interval, with ultrasonography as indicated."

Another example could be if this same palpable mass were solid and probably benign. If the patient is undergoing in vitro fertiliza- tion, then a biopsy would be reasonable. Thus, the radiologist could render a BI-RADS 3 reading and still offer a US-guided biopsy for the diagnosis, if this would help in clinical management. This newly instituted flexibility in language officially sanctions what we already do in our reports, as we fulfill the role of a physician radiologist who appreciates the "big picture" clinical context in which our work matters.

\section{Summary and Conclusion of BI-RADS for US}

The BI-RADS fifth edition includes the second edition for breast US. This long-awaited and eagerly anticipated update comprises changes and additions in the US lexicon with real cases similar to the author's own cases used here for the purpose of illustration. Modifications achieve several overarching goals, namely harmonizing lexicons across modalities (mammography, US, and MRI), anticipating the growing trends of supplemental screening and specialized technologies such as automated breast US and elastography, and helping to guide us towards an effective and efficient interpretation and reporting. Sections on reporting and FAQs are useful for addressing practical questions that may arise in our daily work as breast imagers. The authors of the ACR BI-RADS lexicon do welcome feedback from us, all of us. The lexicon is a living entity and was designed to flex with the times and with our collective needs. Therefore, radiologists should use it well and be empowered to provide the feedback that can help further refine it.

ORCID: Jiyon Lee: http://orcid.org/0000-0003-0036-8828

\section{Conflict of Interest}

Conflict of interest relevant to this article was not reported.

\section{Reference}

1. D'Orsi CJ, Sickles EA, Mendelson EB, Morris EA. ACR BI-RADS atlas, breast imaging reporting and data system. Reston, VA: American College of Radiology, 2013. 